\title{
Saint Petersburg magnetic observatory: from Voeikovo subdivision to INTERMAGNET certification
}

\author{
Roman Sidorov $^{1}$, Anatoly Soloviev ${ }^{1,2}$, Roman Krasnoperov ${ }^{1}$, Dmitry Kudin ${ }^{1,3}$, Andrei Grudnev ${ }^{1,2}$, Yury Kopytenko ${ }^{4}$, \\ Andrei Kotikov ${ }^{4,5}$, and Pavel Sergushin ${ }^{4}$ \\ ${ }^{1}$ Geophysical Center of the Russian Academy of Sciences (GC RAS), 119296 Moscow, Russian Federation \\ ${ }^{2}$ Schmidt Institute of Physics of the Earth of the Russian Academy of Sciences (IPE RAS), 123242 Moscow, \\ Russian Federation \\ ${ }^{3}$ Laboratory of Robot Technique, Gorno-Altaisk State University (GASU), 649000 Gorno-Altaisk, Altai Republic, \\ Russian Federation \\ ${ }^{4}$ Saint Petersburg branch, Pushkov Institute of Terrestrial Magnetism, Ionosphere and Radio Wave Propagation of the \\ Russian Academy of Sciences (IZMIRAN), 199034 Saint Petersburg, Russian Federation \\ ${ }^{5}$ Department of Physics of the Earth, Saint Petersburg State University, 199034 Saint Petersburg, Russian Federation
}

Correspondence to: Roman Sidorov (r.sidorov@gcras.ru)

Received: 15 May 2017 - Discussion started: 5 July 2017

Revised: 26 September 2017 - Accepted: 28 September 2017 - Published: 8 November 2017

\begin{abstract}
Since June 2012 the Saint Petersburg magnetic observatory is being developed and maintained by two institutions of the Russian Academy of Sciences (RAS) - the Geophysical Center of RAS (GC RAS) and the Saint Petersburg branch of the Pushkov Institute of Terrestrial Magnetism, Ionosphere and Radio Wave Propagation of RAS (IZMIRAN $\mathrm{SPb}$ ). On 29 April 2016 the application of the Saint Petersburg observatory (IAGA code SPG) for introduction into the INTERMAGNET network was accepted after approval by the experts of the first definitive dataset over 2015, produced by the GC RAS, and on 9 June 2016 the SPG observatory was officially certified. One of the oldest series of magnetic observations, originating in 1834, was resumed in the $21 \mathrm{st}$ century, meeting the highest quality standards and all modern technical requirements. In this paper a brief historical and scientific background of the SPG observatory foundation and development is given, the stages of its renovation and upgrade in the 21 st century are described, and information on its current state is provided. The first results of the observatory functioning are discussed and geomagnetic variations registered at the SPG observatory are assessed and compared with geomagnetic data from the INTERMAGNET observatories located in the same region.
\end{abstract}

\section{Historical background}

First observations of the Earth's magnetic field elements and their variations in the vicinity of Saint Petersburg date back to 1726 . In 1834 a regularly functioning Russian network for geophysical observations was established (Pasetsky and Svetlaev, 1978). After the Saint Petersburg Main Physical Observatory became the part of the Academy of Sciences, the prominent Russian physicist, chemist, and metrologist Adolph Theodor Kupffer introduced a project of establishing a magnetic and meteorological observatory located outside the city in 1865 . This plan was implemented in 18761878 , and in 1878 the Pavlovsk observatory started functioning on a regular basis. A continuous series of magnetic measurements at the Pavlovsk observatory was maintained from 1878 to 1941 . By the end of World War II the magnetic measurements were continued at the magnetic and meteorological observatory Voeikovo, operated by the Leningrad branch of the Research Institute of Terrestrial Magnetism (currently IZMIRAN).

In the late 1960s a magnetic station Krasnoe Ozero (literally, the Red Lake) was established in the Vyborg district of the Leningrad Region, $100 \mathrm{~km}$ northwest of the city of Leningrad (currently Saint Petersburg) and $89 \mathrm{~km}$ southeast of the city of Vyborg (Fig. 1). This station initially was the branch of the Voeikovo observatory. It was deployed for re- 

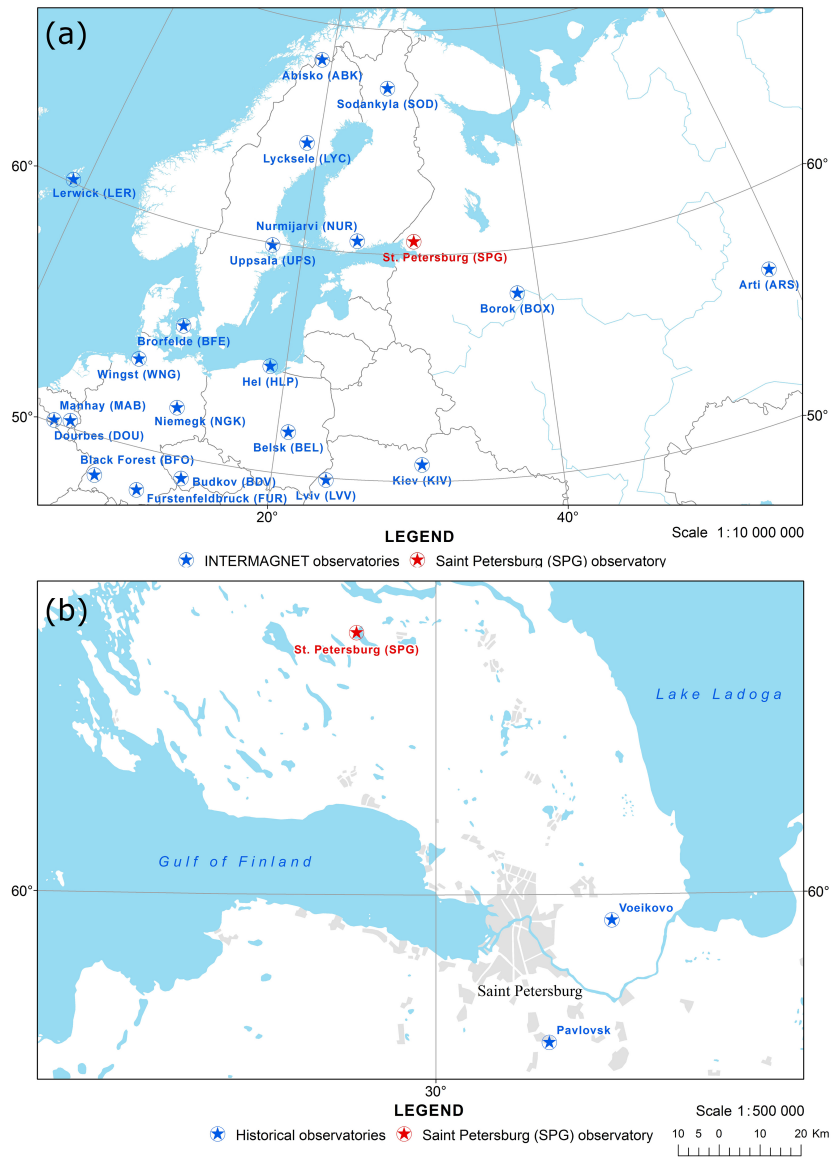

Figure 1. Geographical location of the Saint Petersburg observatory. Current geographical location in relation to other INTERMAGNET observatories (a) and in relation to the historical observatory sites Pavlovsk and Voeikovo (b).

location of magnetometric equipment and instruments from Voeikovo since the level of anthropogenic disturbances had become unacceptable for proper observations. The Krasnoe Ozero station operated until 2000. In 2010, GC RAS and IZMIRAN SPb agreed to deploy a new collaborative highquality magnetic observatory on the basis of the Krasnoe Ozero magnetic station in the course of development of the Russian segment of the INTERMAGNET network (Soloviev et al., 2013; Gvishiani et al., 2014; Gvishiani and Lukianova, 2015). With the joint efforts of these two institutions of the Russian Academy of Sciences this project has become a new milestone in the history of magnetic measurements in this region. This new INTERMAGNET-standard observatory was designated the official name "Saint Petersburg" and IAGA code SPG.

\section{Observatory deployment}

In 2012 the process of renovation of the Krasnoe Ozero magnetic station and deployment of the SPG observatory were initiated.

\subsection{Magnetic survey of the territory}

The SPG deployment was preceded by a detailed magnetic survey of the station's territory for assessment of its magnetic characteristics and detection of possible sources of magnetic disturbances. The survey consisted of measuring the magnetic anomalies and magnetic field vertical gradient. The vertical gradient of the total magnetic field vector was determined as the ratio of difference in readings between two magnetic gradiometer sensors mounted vertically above each other to the distance between the sensors' axes (in most cases it was $56 \mathrm{~cm}$ - the length of one standard rod section between the sensors). Magnetic gradiometry allows to reveal spatially small disturbances against the background anomalies related to geological inhomogeneities. A gradiometer (GEM Systems GSM-19GW) also provides elimination of time variations of the field during the survey, estimating only spatial effects. In addition, the usual modification of a GEM Systems GSM-19 scalar magnetometer was installed as a base station for compensation of the magnetic field diurnal variations that could affect the survey interpretation.

The magnetic survey points within a $4100 \mathrm{~m}^{2}$ area surrounding the pavilions were set out for the magnetic survey using an optical theodolite and a $50 \mathrm{~m}$ geodetic measuring tape. The overall length of survey lines was $480 \mathrm{~m}$ (56 magnetic survey points) with a spatial resolution of $10 \times 10 \mathrm{~m}$, as the interval between the survey points and the distance between the survey lines was $10 \mathrm{~m}$. This was considered as an optimal spatial resolution to reveal possible heterogeneities the distribution of anomalies of the total magnetic field and its vertical gradient. The overall error of setting out the survey points was $\sim 20 \mathrm{~cm}$, i.e., $2 \%$ of the spatial resolution of the survey, which was considered negligible. The survey lines were marked along a north-south direction. The builtin global navigation satellite system (GNSS) receiver of the gradiometer was used to determine the survey point coordinates. After the survey, the measurements were processed on a PC, where the recorded data were imported from the magnetic gradiometer and the base magnetometer. Time variations (diurnal variation, pulsations, etc.), occurring during the survey were compensated while processing. For this purpose, the gradiometer data recordings corresponded in time with the ones recorded by the base magnetometer, so that the time of the registration for every recording of the magnetic gradiometer coincided with or was nearest to the time of the registration for the base magnetometer data. Thus, the anomalous component $\Delta F_{\mathrm{a}}$ of the total magnetic field intensity at each observation point was calculated using the fol- 
lowing Eq. (1):

$\Delta F_{\mathrm{a}}(i)=F_{\mathrm{s}}\left(t_{\mathrm{s}}(i)\right)-F_{\mathrm{b}}\left(t_{\mathrm{b}}(j)\right)$,

where $F_{\mathrm{s}}$ is the survey magnetic recording, $F_{\mathrm{b}}$ is the base magnetometer recording, and the time moment of a survey data recording $t_{\mathrm{S}}(i)$ and the one of a base magnetometer recording $t_{\mathrm{b}}(j)$ produce the minimum of the difference $\left|t_{\mathrm{S}}(i)-t_{\mathrm{b}}(j)\right|$ (including zero if they match second to second). The GSM-19 at the absolute pavilion was used as a base magnetometer; its sampling rate was $3 \mathrm{~s}$.

After that the maps of the anomalous component and the vertical gradient of total field were plotted. We used triangulation with linear interpolation for gridding the survey data along a regular grid.

The map analysis showed that the territory surrounding the pavilions was generally characterized by homogeneous field. A strong magnetic anomaly to the west of the $\Delta F_{\mathrm{a}}$ plot (Fig. 2a) results from certain gardening equipment which did not affect the overall magnetic field distribution significantly (later the source of anomaly was removed to provide even more magnetic cleanliness). The lateral spatial variability of the magnetic field was considered insignificant in the vicinity of the pavilions. The distribution of the vertical gradient values over the survey plot (Fig. 2b) is represented mainly by zero values. Therefore, the results of the survey showed that the area of the Krasnoe Ozero station was suitable for the installation of the INTERMAGNET-standard equipment for registering the total magnetic field and its variations and for carrying out absolute measurements. During this stage preliminary absolute measurements were performed as well to estimate the characteristic values of magnetic declination and inclination within the station's site.

\subsection{Observatory infrastructure renovation}

By the end of 2012 the interior of the station's pavilions was renovated (Fig. 3), and a new heating system was installed. The marble instrument pillars which existed at the observatory from the beginning were repaired, and their tops were fixed using grout. Initially the Krasnoe Ozero station had a water heating system based on water supply via copper pipelines. Currently this system is not used. In the absolute pavilion, a demountable wooden screen with an illuminator was placed over the window for directed sighting the azimuth mark. This helps to regulate the thermal balance and avoid refraction due to the temperature contrast when opening the window in cold seasons.

\subsection{Installation of magnetometric instruments}

In 2012 a full set of magnetometric instruments of the INTERMAGNET standard was installed into the observatory pavilions. It includes a DTU Space FGE vector fluxgate magnetometer, a GEM Systems GSM-19 proton scalar Overhauser magnetometer, a Mingeo fluxgate declinome- ter/inclinometer based on Carl Zeiss Theo010 non-magnetic theodolite, and a Mingeo Magrec data acquisition system.

The DTU Space FGE vector fluxgate magnetometer was installed on a pillar at the variation pavilion. This magnetometer is equipped with a 24 bit $\mathrm{AD}$ converter and provides digital registration of measurements at the frequency up to $10 \mathrm{~Hz}$. Positioning and adjusting of the magnetometer's sensor system with reference to the geographic coordinate system was considered more preferable. Although the process of the sensors' alignment in the magnetic coordinate system is often recommended by various researchers as a relatively easy alternative, it requires future recurrent adjustments of the sensor direction due to variability of the magnetic North Pole coordinates. Thus, the measured values for the vector magnetometer correspond to the variations of the magnetic field components in the northern $(X)$, eastern $(Y)$, and vertical $(Z)$ geographical directions. The absolute values of the magnetic field vector components were calculated based on the performed absolute observations.

The GEM Systems GSM-19 scalar magnetometer sensor was mounted on the top of a pillar inside the absolute pavilion in a wooden cradle and fixed. The GSM-19 sensor axis was oriented horizontally and normal to the magnetic meridian plane. The fluxgate declinometer/inclinometer was mounted on another pillar in the absolute pavilion. We defined the difference of the total field between the absolute measurement pillar and the pillar where the scalar magnetometer sensor was mounted. This difference was $5.5 \mathrm{nT}$, and it was most likely caused by some elements of the pavilion interior (such as power cables) or some natural disturbances. The determination of the pillar total field difference was repeated in 2014 or 2015, and the difference value appeared to be generally the same except for some second decimal places, which indicates the stability of the magnetic environment at the observatory site.

The observatory data acquisition system, installed in the main building, was configured for transmission of data in the near-real-time mode to GC RAS, IZMIRAN, and later to the INTERMAGNET GIN in Paris. Low-voltage (12 V) batteries supply power to the scalar, vector magnetometers, and the Magrec data acquisition system via underground power lines. The lighting protection modules were installed to prevent possible damages of power lines due to their overvoltage from thunderstorms.

In 2015 a series of improvements in data transmission were implemented by GC RAS specialists. A new antenna for amplification the $3 \mathrm{G}$ internet connection was mounted, and a new $3 \mathrm{G}$ modem was installed directly into the Magrec data logger. The latter was set up to provide remote access and control. Also, in autumn 2015 certain improvements were made to achieve a better data quality. First, the sources of distortion of the magnetic records from the FGE vector magnetometer were eliminated. Next, the total check of electric connections, hardware cables, and the heating sys- 


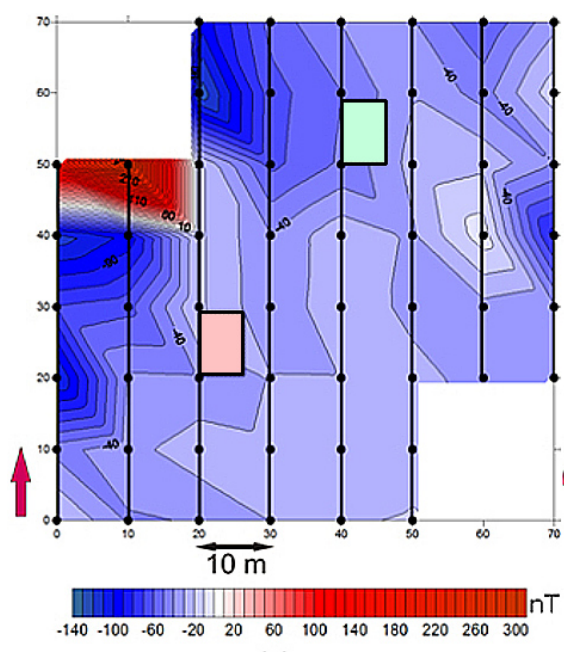

(a)

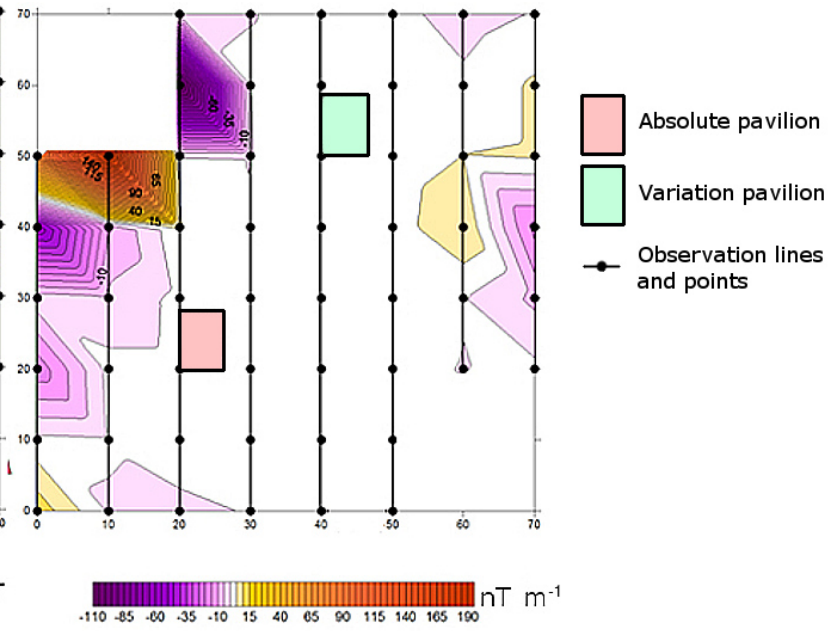

(b)

Figure 2. Maps of magnetic anomalies (a) and vertical magnetic gradient (b) on the observatory territory.

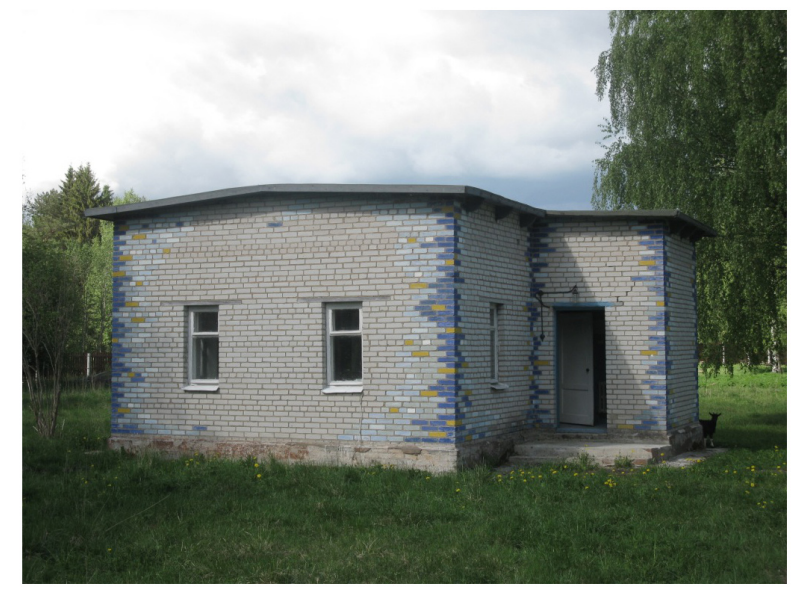

(a)

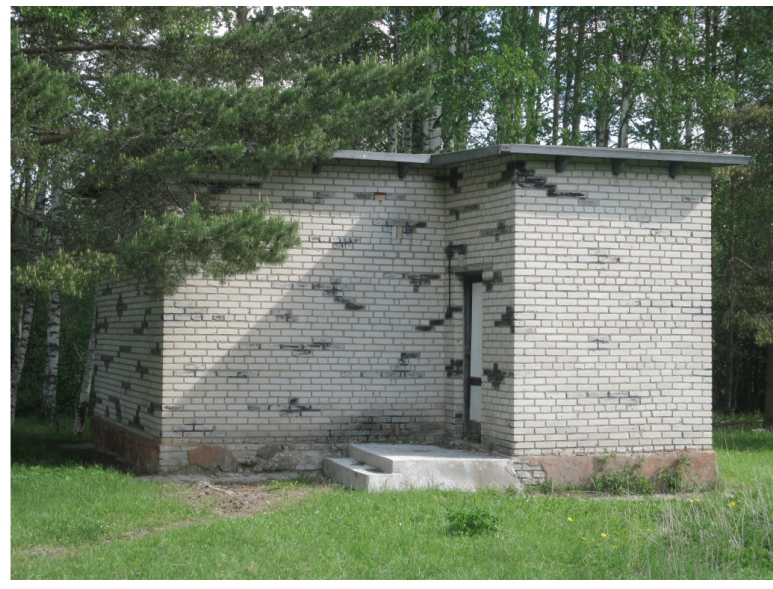

(b)

Figure 3. Absolute (a) and variation (b) pavilions of the Saint Petersburg observatory.

tem was done at the variation pavilion. Finally, the software for the data acquisition system computer was updated.

\subsection{Azimuth mark installation and reference azimuth determination}

In 2012 a new observatory azimuth mark (or mira) was constructed. The requirements for installing the reference azimuth mark for absolute measurements are significantly important since the measurements of magnetic declination and inclination require direct visibility of the azimuth mark or a remote benchmark. As it is commonly not possible to use a fundamental structure in $1-2 \mathrm{~km}$ from the absolute pavilion as an azimuth mark, one should consider that the shorter the distance between the mark and the observation point, the firmer it should be fixed. For example, the shift of the az- imuth mark installed in $100 \mathrm{~m}$ from the observation point should not be more than $3 \mathrm{~mm}$ laterally.

The azimuth mark for the SPG observatory was developed, assembled and tested at the Voeikovo observatory and installed at SPG in autumn 2012. It is a steel construction equipped with a light bulb for carrying out the absolute measurements in case of possible poor visibility and lack of light due to weather conditions. The mark is mounted on four supports onto a concrete basement providing high stability (Fig. 4a). The mark can be clearly seen from the measurement pillar through the theodolite telescope (Fig. 4b).

In the manuals dedicated to ground magnetic observatory practice (Jankowski and Sucksdorff, 1996; Nechaev, 2006), it is recommended to determine the reference azimuth for absolute measurements by carrying out astronomical observations. Azimuth determination from Sun observations en- 

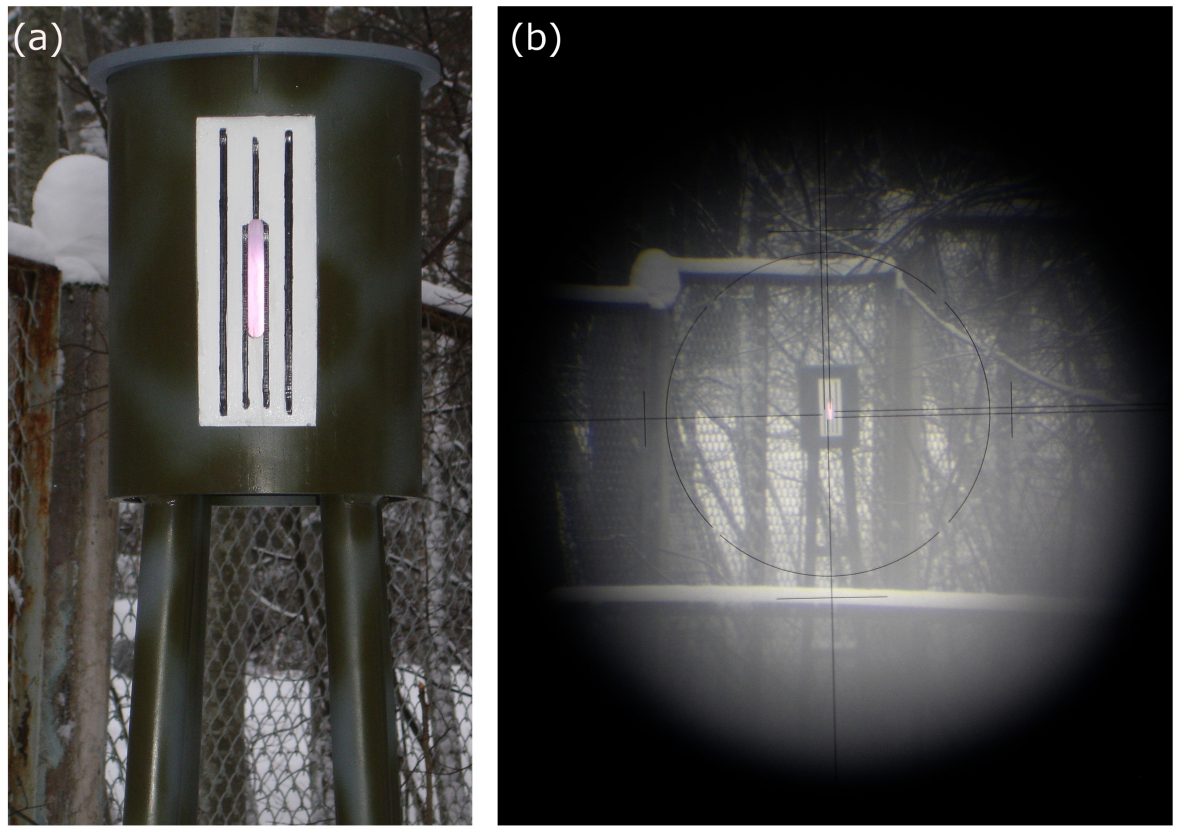

Figure 4. Reference azimuth mark. The light bulb compartment (a) and the view of the mark from the absolute pavilion (b).

sures an astronomical azimuth value with an error of about dozens of arcseconds. However, such an approach is significantly labor intensive and also requires high-accuracy geodetic tools and a qualified operator. With the development of GNSS technologies, it became possible to determine the reference direction at magnetic observatories without the mentioned disadvantages. A method of determining coordinates was recommended in a guide (Newitt et al., 1996). It provides the positioning of a station within a $2 \mathrm{~cm}$ accuracy and determining of geodetic azimuths of reference directions on distances of several hundred meters with an accuracy of several arcseconds. The accuracy of azimuth determination depends on the length of the baseline: the longer the baseline direction is, the higher the accuracy of the reference azimuth measurement. This method has already been implemented at magnetic repeat stations abroad (Lalanne et al., 2013) but it has not yet become a wide practice at geomagnetic observatories in Russia. The approach for azimuth determination of reference directions at magnetic observatories, based on modern geodetic equipment and technologies, has been successfully introduced and implemented by the GC RAS' specialists (Kaftan and Krasnoperov, 2015; Krasnoperov et al., 2015).

The geodetic equipment that was used for the measurements included two sets of GPS Javad Maxor receivers with Legant antennae and a Trimble M3 DR 5" electronic laser total station with a standard prism reflector. The GPS receivers were positioned at auxiliary points for determining the azimuth of the baseline between these points. Point positioning was performed in differential mode. Two continuously operating GPS stations SVTL and PULK, located within $150 \mathrm{~km}$ from the observatory site, were used as base stations. The total station and prism reflectors were used to transmit the geodetic azimuth to the reference direction of the mark. In the conditions of forestation and other obstacles for mutual visibility between the points on the territory of the SPG observatory, it was impossible to obtain a baseline longer than $150 \mathrm{~m}$; however, the accuracy of determining the azimuth of the reference direction was 2-3 arcsec (Kaftan and Krasnoperov, 2015), which meets the INTERMAGNET requirements for the azimuth mark given in Benoit (2012), Jankowski and Sucksdorff (1996), and Newitt et al. (1996). Also, the coordinates of the pillar centers at the absolute pavilion of the observatory were obtained for the first time with reference to the common international coordinate reference frame.

\section{Observatory data analysis and discussion}

\subsection{Variation data analysis}

To make sure that the correct functioning of the magnetometers and proper variation data quality is provided, we made a qualitative and quantitative comparison between the data records registered at the SPG observatory and the ones from the Borok (BOX, Russia), Lerwick (LER, UK), Nurmijarvi (NUR, Finland), and Uppsala (UPS, Finland) observatories. These four INTERMAGNET observatories are the closest to the SPG observatory both by geographic and magnetic latitudes (see Table 1 for details on their geographic locations). We selected three time periods corresponding to different space weather and solar activity conditions to es- 
timate the geomagnetic variation signal forms during quiet and disturbed periods, as it can provide a more representative overview of the magnetometers' operation compared to the ones at other observatories.

The periods were chosen for this research according to the Kp index data (Kp-Index - Helmholtz Centre Potsdam GFZ German Research Centre for Geosciences, 2017). We chose two disturbed periods in March and June 2015 to estimate the response of the magnetometers to two geomagnetic storms occurred during the period that later was selected for the INTERMAGNET certification of the SPG observatory. On 1718 March 2015 the strongest geomagnetic storm of the current solar cycle took place. During this storm the planetary Kp index values varied from $4+$ to $8-$, and the Kp sum was 480 for the first day of the storm and $39+$ for the second day (here we use common notations for Kp magnitudes). Its analysis based on SPG data is discussed in A. D. Gvishiani et al. (2016). Another storm occurred on 22-24 June 2015, and it was also a large geomagnetic activity disturbance: the $\mathrm{Kp}$ values varied from $3+$ to $8+$, and the $\mathrm{Kp}$ sum was $35+$ for 22 June and $42-$ for 23 June. We also compared the geomagnetic records for a quiet geomagnetic activity period and selected the INTERMAGNET data available for such period in June 2016. We considered a period 1-4 June 2016 represented by Kp index values within the range of $0 \mathrm{o}$ to $2 \mathrm{o}$.

The horizontal components of the total field vector are commonly the most affected by magnetic disturbances, so in this paper we demonstrate the comparison plots only for the $X$ component as the most illustrative. The data plots for the mentioned periods are given in Figs. 5-7. It is clearly seen that the SPG vector magnetometer records for the disturbed periods generally match the ones for these periods from the other observatories. Maximal signal similarity for $X$ records can be seen between SPG (Figs. 5a and 6a) and BOX (Figs. $5 \mathrm{~b}$ and $6 \mathrm{~b}$ ) as well as between SPG and NUR (Figs. 5d, 6d). The similarity between BOX and LER (Figs. 5c and 6c) is not so obvious. Probably this is due to the location of the LER observatory and its geomagnetic latitude $\left(61.67^{\circ} \mathrm{N}\right.$ for 2015 and $61.65^{\circ} \mathrm{N}$ for 2016), which is higher than the geomagnetic latitude for other two observatories from our list (about $\left.53-57^{\circ} \mathrm{N}\right)$, although geographical latitude $\left(60.13^{\circ} \mathrm{N}\right)$ is very close to the one for SPG $\left(60.542^{\circ} \mathrm{N}\right)$. The difference in geomagnetic latitude means the difference in geomagnetic conditions due to a higher impact of auroral oval dynamics in the region of the observatory location. However, it is still possible to visually match the signal fragments between SPG and LER $X$ components corresponding to storm sudden commencements, onsets, main phases, and the start moments of recovery phase. The data plots for quiet geomagnetic conditions (1-4 June 2016) again demonstrate high signal similarities between SPG (Fig. 7a) and BOX (Fig. 7b) and between SPG and NUR (Fig. 7d) variation data and lower similarity between SPG and LER (Fig. 7c) data. Here we do not provide the similar plots for the comparison between SPG and
UPS $X$ component as they generally repeat the plots for SPG and NUR being close latitudinally.

For a quantitative assessment of the data similarity we estimated the Pearson linear correlation coefficient between SPG $X, Y, Z$, and $F$ records and the corresponding records from other four observatories during the abovementioned time periods. We also included the Uppsala (UPS) magnetic observatory, The correlation coefficient values are listed in Table 2. The correlation values prove the visual similarities of the data plots. Maximum correlation coefficient values are between SPG and UPS observatory (up to 1.00 for $X$ component) and NUR observatory (0.96-0.99). No scalar data for NUR observatory were available during all the analyzed periods. Also high correlation values were obtained for SPG and BOX observatories. The comparison for SPG and LER observatories resulted in the lowest correlation coefficient values, though still indicating a strong positive correlation $(0.65-0.72)$. Hence, the quantitative comparison again shows the highest similarity between SPG and NUR observatory data due to their geographical and geomagnetic latitudinal closeness. Data records for another geomagnetically close observatory - BOX - also have similarities, and the variation data from LER observatory have some differences comparing to SPG data because of differences in geomagnetic location and, therefore, differences in geomagnetic conditions. Probably the same is true for the correlation between the $Y$ component data from SPG and UPS which appeared to be lower than the one for other components during the stormy periods $(0.76-0.78)$.

Thus, the results of the comparison between the variation data from the SPG observatory and the corresponding variation datasets from three abovementioned geographically close INTERMAGNET observatories generally confirm that the magnetic equipment of the observatory is correctly installed, the vector magnetometer is properly aligned in the geographical coordinate system, and there are no significant sources of regular electromagnetic noise in the vicinity of the observatory site.

As an additional check of the quality of the variation recordings, we used the data from the POS-4 Overhauser vector magnetometer. This device was manufactured by the Laboratory of Quantum Magnetometry of Ural State Technical University (USTU-UPI, Yekaterinburg, Russia) and allows a simultaneous recording of horizontal, vertical, and total field component values at high absolute precision. POS-4 magnetometers proved its long-term stability during the test measurements at Russian INTERMAGNET magnetic observatories Arti (ARS) and Paratunka (PET) (Sapunov et al., 2016). In June 2017 the POS-4 magnetometer was installed by the GC RAS temporarily at the SPG observatory in a small supplementary pavilion. The first datasets registered by POS-4 magnetometer are displayed in Fig. 8. A significant agreement between the $Z$ absolute component recordings from POS- 4 and the quasi-definitive $Z$ data derived from the variations from FGE vector magnetometer (Fig. 8a, b), as 
Table 1. Reference information on the observatories whose variation records were chosen to be compared with the Saint Petersburg observatory data.

\begin{tabular}{llllllll}
\hline IAGA code & $\begin{array}{l}\text { Observatory name, } \\
\text { country }\end{array}$ & Geogr. lat & Geogr. long & $\begin{array}{l}\text { Mag. lat } \\
\text { for 2015 }\end{array}$ & $\begin{array}{l}\text { Mag. long } \\
\text { for 2015 }\end{array}$ & $\begin{array}{l}\text { Mag. lat } \\
\text { for 2016 }\end{array}$ & $\begin{array}{l}\text { Mag. long } \\
\text { for 2016 }\end{array}$ \\
\hline BOX & Borok, Russia & $58.068^{\circ} \mathrm{N}$ & $38.233^{\circ} \mathrm{E}$ & $53.43^{\circ} \mathrm{N}$ & $123.53^{\circ} \mathrm{E}$ & $53.45^{\circ} \mathrm{N}$ & $123.56^{\circ} \mathrm{E}$ \\
LER & Lerwick, United Kingdom & $60.13^{\circ} \mathrm{N}$ & $1.18^{\circ} \mathrm{W}$ & $61.67^{\circ} \mathrm{N}$ & $88.63^{\circ} \mathrm{E}$ & $61.65^{\circ} \mathrm{N}$ & $88.64^{\circ} \mathrm{E}$ \\
NUR & Nurmijarvi, Finland & $60.51^{\circ} \mathrm{N}$ & $24.66^{\circ} \mathrm{E}$ & $57.79^{\circ} \mathrm{N}$ & $112.99^{\circ} \mathrm{E}$ & $57.80^{\circ} \mathrm{N}$ & $113.00^{\circ} \mathrm{E}$ \\
UPS & Uppsala, Finland & $59.900^{\circ} \mathrm{N}$ & $17.400^{\circ} \mathrm{E}$ & $58.36^{\circ} \mathrm{N}$ & $106.11^{\circ} \mathrm{E}$ & $58.36^{\circ} \mathrm{N}$ & $106.12^{\circ} \mathrm{E}$ \\
SPG & Saint Petersburg, Russia & $60.542^{\circ} \mathrm{N}$ & $29.716^{\circ} \mathrm{E}$ & $57.06^{\circ} \mathrm{N}$ & $117.42^{\circ} \mathrm{E}$ & $57.07^{\circ} \mathrm{N}$ & $117.44^{\circ} \mathrm{E}$ \\
\hline
\end{tabular}

Table 2. Correlation coefficients for the magnetic field components from SPG and other observatories during the periods of disturbed (17-18 March and 22-24 June 2015) and quiet magnetic field (1-4 June 2016).

\begin{tabular}{|c|c|c|c|c|c|c|c|c|c|c|c|c|}
\hline \multirow[t]{3}{*}{ IAGA code } & \multicolumn{12}{|c|}{ Correlation coefficient between the component records } \\
\hline & \multicolumn{4}{|c|}{$\begin{array}{l}\text { 17-18 Mar } 2015 \\
\text { (storm period) }\end{array}$} & \multicolumn{4}{|c|}{$\begin{array}{l}\text { 22-24 Jun } 2015 \\
\text { (storm period) }\end{array}$} & \multicolumn{4}{|c|}{$\begin{array}{l}\text { 1-4 Jun } 2016 \\
\text { (quiet period) }\end{array}$} \\
\hline & $X$ & $Y$ & $Z$ & $F$ & $X$ & $Y$ & $Z$ & $F$ & $X$ & $Y$ & $Z$ & $F$ \\
\hline BOX & 0.91 & 0.78 & 0.67 & 0.82 & 0.92 & 0.83 & 0.77 & 0.85 & 0.94 & 0.98 & 0.88 & 0.93 \\
\hline LER & 0.86 & 0.61 & 0.51 & 0.72 & 0.83 & 0.49 & 0.47 & 0.69 & 0.72 & 0.71 & 0.65 & 0.78 \\
\hline NUR & 0.99 & 0.89 & 0.81 & - & 0.99 & 0.89 & 0.95 & - & 0.99 & 0.99 & 0.96 & - \\
\hline UPS & 0.94 & 0.78 & 0.66 & 0.84 & 0.95 & 0.76 & 0.92 & 0.95 & 1.00 & 0.93 & 0.95 & 0.92 \\
\hline
\end{tabular}

well as the agreement between the total field recordings from POS-4 and from GSM-19 scalar magnetometer (Fig. 8c, d), is clearly seen (the spikes on POS-4 records are of anthropogenic origin). The differences between the POS-4 data, resampled to $1 \mathrm{~min}$ rate, and the quasi-definitive observatory data are close to constant for $Z$ (Fig. 8e) and for $F$ (Fig. 8f) components.

Next, let us discuss the first definitive dataset for the SPG observatory as the main and final proof of its quality and suitability for the INTERMAGNET network in all aspects concerning the requirements formulated for its magnetic observatories (Benoit, 2012).

\subsection{Absolute, baseline, and definitive data analysis}

The most important problem of magnetic observatories is recording the Earth's magnetic field secular variation over long time periods (decades, centuries, and even longer periods). To date, vector measurements of magnetic variations using fluxgate magnetometers similar to DTU Space FGE (Denmark) and IPGP VM391 (France) are carried out at many observatories. These vector variation measurements possess an unavoidable and unpredictable (Chulliat and Anisimov, 2008) drift on such long intervals mainly because of temperature variations, pillar and sensor deterioration, and instrument aging. Therefore, there is a need to perform regular measurements of the absolute magnetic declination, inclination and total intensity with a fluxgate magnetometer mounted on a non-magnetic optical theodolite (DI- flux magnetometer) and a scalar magnetometer for vector data calibration (Jankowski and Sucksdorff, 1996).

Regular absolute measurements started at the SPG observatory in 2013 and they are taken at least once a week. The adopted baseline values are calculated for the $X, Y$, and $Z$ components by the algorithm of fitting the spline curve to the observed baseline values resulted from the absolute measurements. Both the observed and adopted baselines in 2015 are presented in Fig. 9. Two baseline jumps in January and February 2015 were due to some improvement and repair works at the observatory. The quality of absolute measurements was estimated by calculating the root-meansquare (RMS) deviation of differences between observed and adopted baseline values for each component. Evident absolute observation outliers were eliminated when spline approximation was applied. The 2015 definitive dataset was prepared at the Russian-Ukrainian Geomagnetic Data Center (2017) with the use of special software toolkit designed as a part of the automated hardware and software multifunctional system for geomagnetic monitoring (A. Gvishiani et al., 2016). The reliability of adopted baseline application can be also estimated by comparing the obtained absolute measurements of the field components and baselinecorrected $1 \mathrm{~min}$ fluxgate recordings taken for the corresponding time moments. The corresponding differences were calculated; their dispersion plots are given in Fig. 10a-c. As can be seen, some outlier values produced the large differences varying from -7.98 to $18.24 \mathrm{nT}$, from -7.09 to $15.86 \mathrm{nT}$, and from -0.78 to $4.23 \mathrm{nT}$ for $X, Y$, and $Z$ components, respectively. After the removal of the outliers, the obtained 

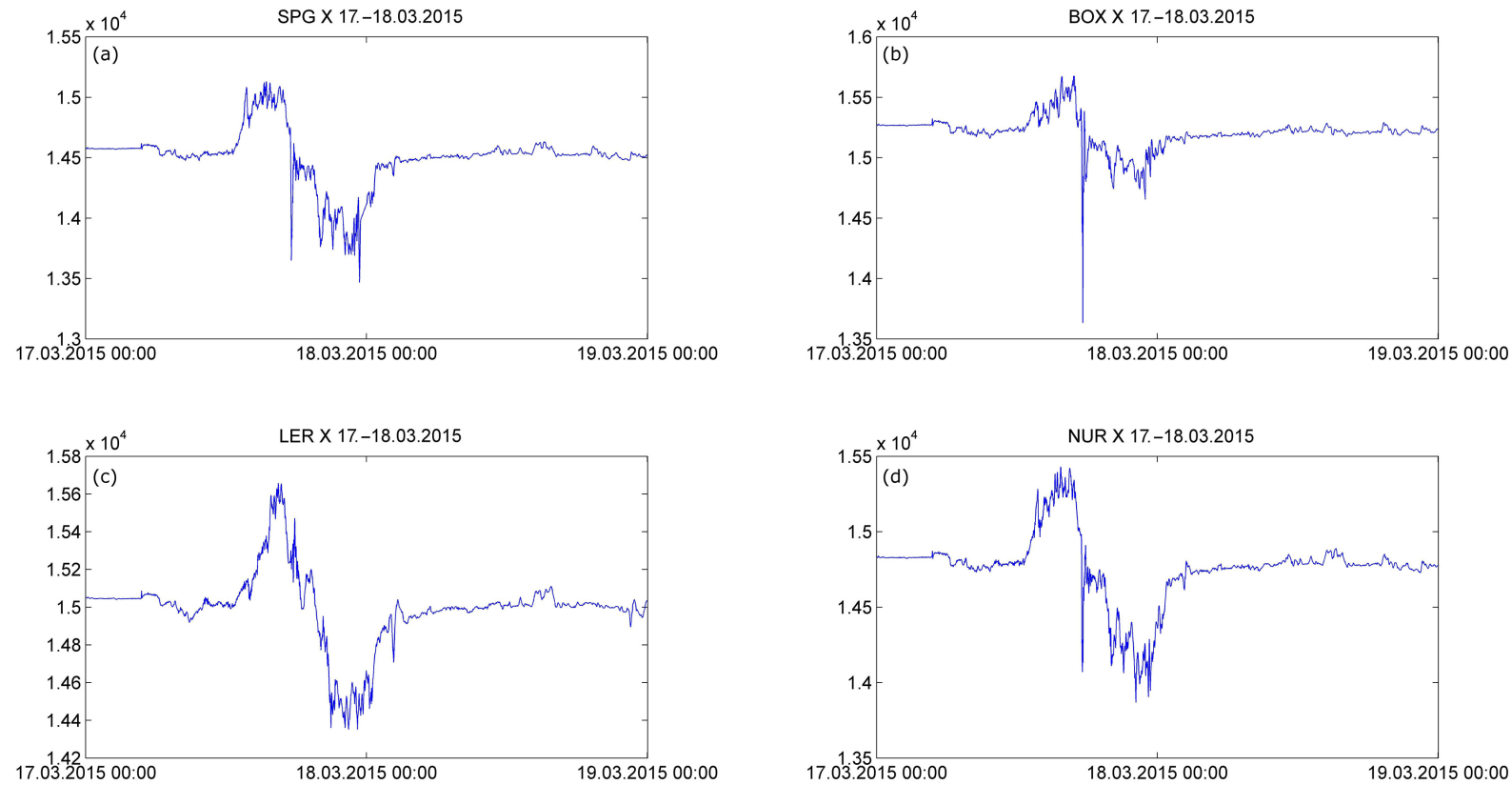

Figure 5. Minute magnetic variations of the $X$ component during the strong magnetic storm of 17-18 March 2015.
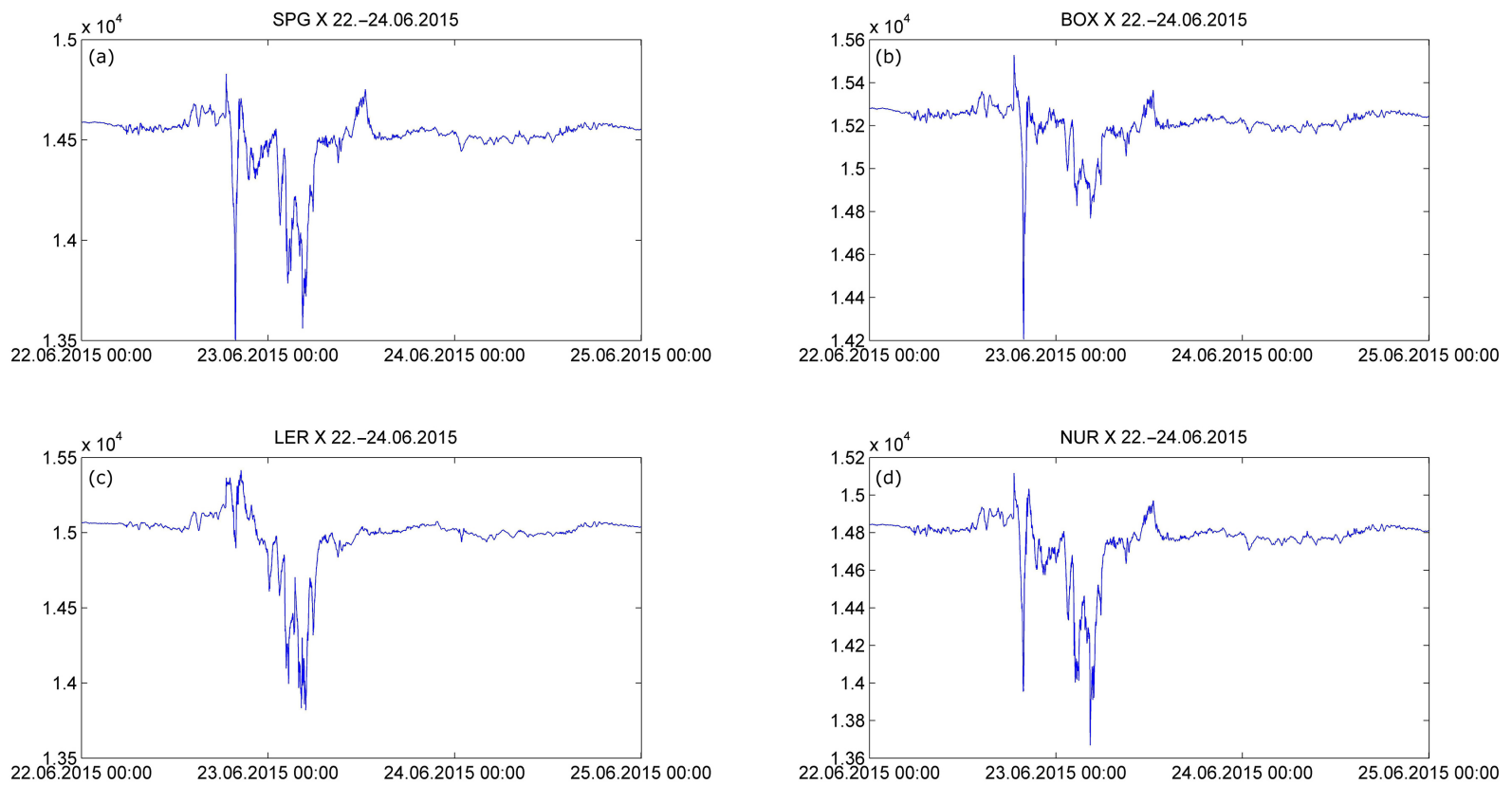

Figure 6. Minute magnetic variations of the $X$ component during the magnetic storm of 22-24 June 2015.

RMS deviations for baselines for the period 1 January 20151 January 2016 were $2.91,2.08$, and $0.61 \mathrm{nT}$ for $X, Y$, and $Z$, respectively.

Also the $\Delta F$ differences were analyzed to confirm 2015 definitive data quality. The $\Delta F$ values are the differences between the total field vector magnitudes directly measured with a scalar magnetometer and calculated from the vector measurements after their correction, using the adopted base- lines. The complete $\Delta F$ series over 2015 is given in Fig. 10d. It is seen that the $\Delta F$ values variability is within $5 \mathrm{nT}$ during a year, which meets the INTERMAGNET requirements (Benoit, 2012).

Finally, we compared definitive values of magnetic field components averaged over several magnetically quiet periods (1-2 January and 3-7 February 2015) with those derived from the internal magnetic field models. We used freely 

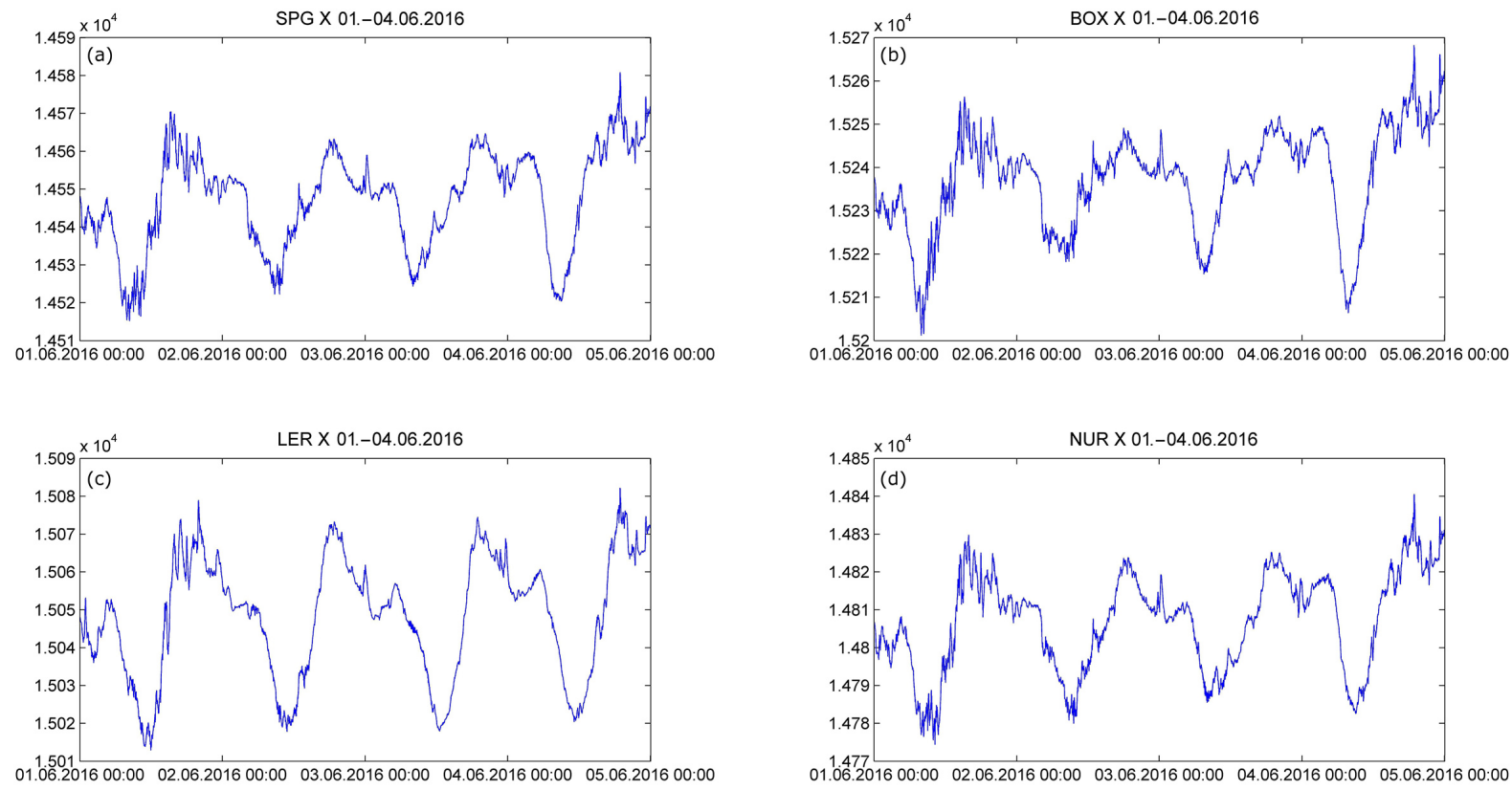

Figure 7. Minute magnetic variations of the $X$ component during a quiet period 1-4 June 2016.

Table 3. Results of comparison between the average absolute values of the magnetic field vector components and the model values for different internal field models. CF stands for core field, and AF for the anomalous (lithospheric) field; the number after the AF in the model name indicates its spherical expansion order and degree.

\begin{tabular}{lcccc}
\hline Model & Epoch & $X$ & $Y$ & $Z$ \\
\hline CHAOS-6 (CF) & 2015.1 & 14353.15 & 2615.35 & 50420.77 \\
CHAOS-6 (CF, AF85) & 2015.1 & 14424.07 & 2672.95 & 50293.85 \\
SIFM (CF) & 2015.1 & 14355.23 & 2616.28 & 50419.06 \\
EMM2015 (CF, AF720) & 2015.0 & 14513.75 & 2648.01 & 50346.38 \\
SPG definitive values & 2015.1 & 14542.02 & 2551.72 & 50260.61 \\
SPG definitive values & 2015.0 & 14543.68 & 2546.5 & 50262.27 \\
\hline
\end{tabular}

available and widely used models, which are considered to be the most precise at the moment: CHAOS-6 (Finlay et al., 2016), SIFM (Olsen et al., 2015), and EMM2015 (Magnetic Crustal Field Model, 2017). All of them are constructed using the high-precision new-generation satellite observations. Some of them include both the contributions of main and lithospheric field (see Table 3). CHAOS-6 and SIFM were used to calculate components for the 2015.1 epoch, and EMM2015 was used to calculate components for the 2015.0 epoch, as it is the upper time limit for the latter model. All the model values were calculated for the SPG observatory geodetic location; local geomagnetic coordinates were reduced to local nominally geodetic coordinate system of magnetic measurements at the observatory. The comparison results generally indicate that the observed and modeled component values are in good agreement. Though the differences between the model values and the observed ones are sometimes more than $100 \mathrm{nT}$, it is still possible to conclude that the magnetometer set is properly installed and the measured and model data are physically close, as the values are close in general (thousands of $\mathrm{nT}$ ). The differences in hundreds of nT can be caused by spatial smoothing, applied in spherical harmonic analysis models, and crustal field bias, not considered in the corresponding models.

\section{Conclusions}

The SPG observatory was founded as a subdivision of the Voeikovo observatory, and in the beginning of the 21 st century it became an autonomous scientific facility providing continuous geomagnetic measurements fully meeting the highest international quality standards. Qualitative and quantitative comparison between the SPG observatory variation and total field data with the data from three other INTERMAGNET observatories (BOX, LER, and NUR), which are located close to it, was done. The comparison demonstrated 


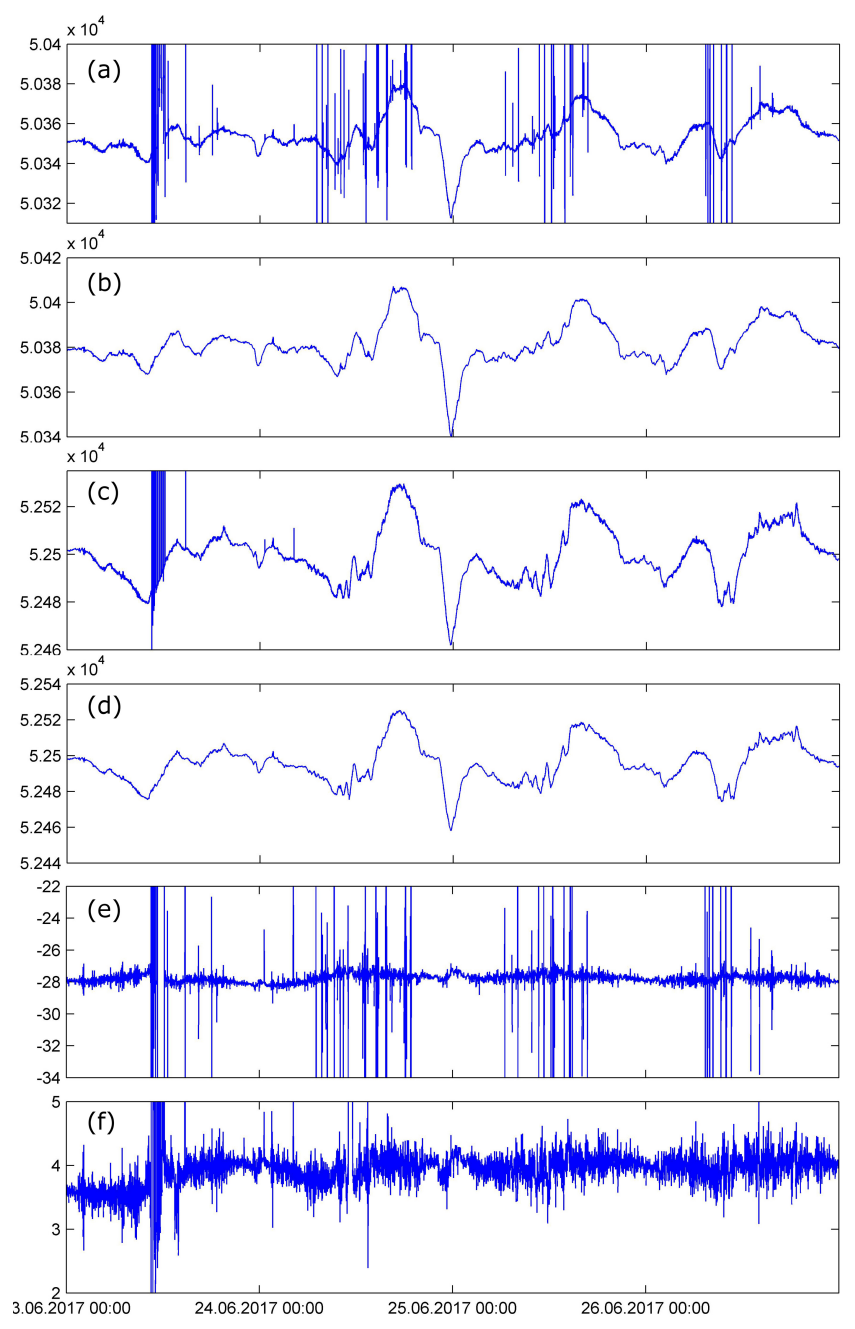

Figure 8. Comparison between the data from POS-4 and the observatory magnetometers: comparison of the $Z$ data between POS-4 (a) and FGE (b) magnetometers; comparison of the total field $F$ data between POS-4 (c) and GSM-19 (d) magnetometers; differences between the $Z$ (e) and $F$ (f) records.

the signal form similarity and relatively high correlation coefficients between the SPG and the closest NUR observatory data both for quiet and disturbed periods. This indicates that the vector magnetometer at the SPG observatory is properly installed and aligned and the data are not affected by regular anthropogenic disturbances. Analysis of definitive data calculated from the SPG observatory variation and absolute data over 2015 using automated software, integrated into the GC RAS's hardware and software system for geomagnetic monitoring (A. Gvishiani et al., 2016), showed that the resulting data records meet the INTERMAGNET standards.

Previously, the Russian INTERMAGNET segment included nine magnetic observatories: Arti, Borok, Irkutsk, Khabarovsk, Magadan, Novosibirsk, Paratunka, Yakutsk, and Vostok. The SPG observatory, recently accepted into the INTERMAGNET network, now also provides $1 \mathrm{~s}$ mag-

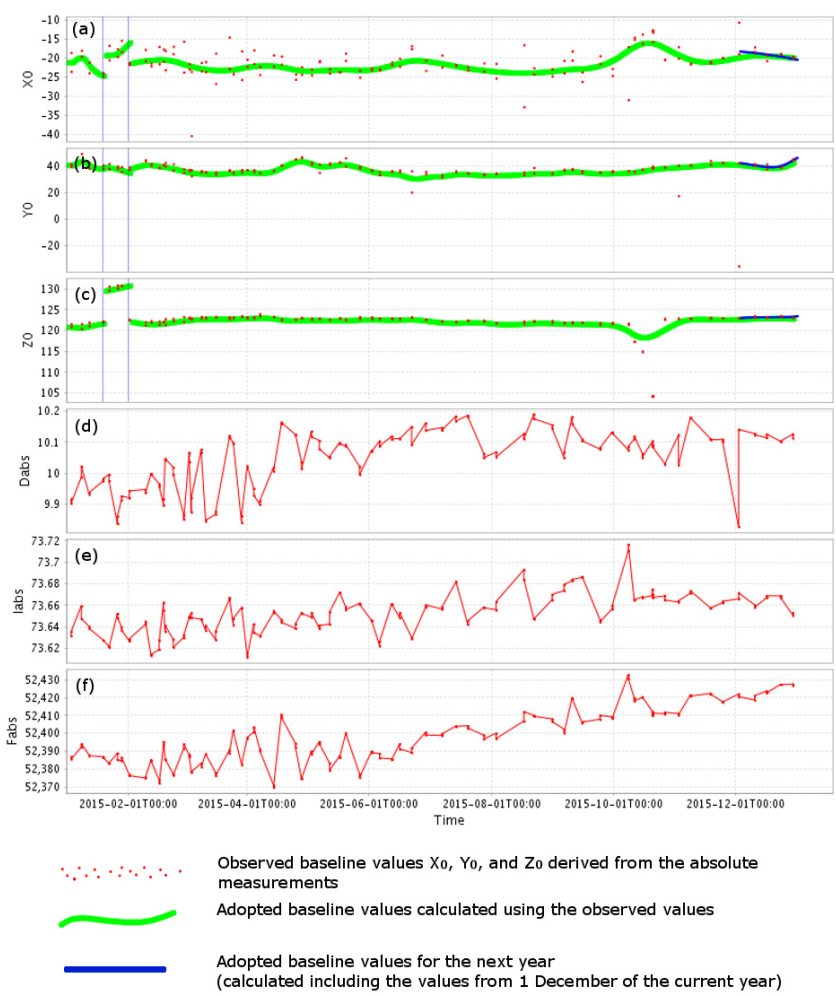

Figure 9. Observed and adopted baseline values (a, b, c) for 2015 and absolute declination (d), inclination (e) and total intensity (f).

netic data with a near-real-time transmission. The inclusion of this new observatory into the INTERMAGNET network contributes to accurate modeling of rapid and longterm variations of the core magnetic field and analysis of geomagnetic disturbances caused by the external magnetic field, their dynamics, and spatiotemporal features. A particular advantage of the SPG observatory is its high-latitude location. Auroral and subauroral regions are mostly subjected to space weather, and therefore magnetic observatories located in these regions are the most valuable source of information for monitoring and forecasting the space weather effects at the Earth's surface. High-latitude INTERMAGNETstandard observatories provide physically reliable datasets for detailed analysis of interplanetary environment and, thus, can be used for calibrating other types of ground-based observation systems, such as neutron monitors and muonic hodoscopes. In particular, recent studies demonstrate that observatory records along with neutron monitor data and muonic hodoscope images can be used in the development of ground-based distant techniques for early warning of geomagnetic storms (Hafez and Ghamry, 2013; Wu and Lundstedt, 1997).

Digital object identifiers were assigned to the whole observatory database (Soloviev, 2016) and to its preliminary (Soloviev et al., 2016) and 2015 definitive (Soloviev et al., 2016) datasets. These data are considered as fully valid re- 

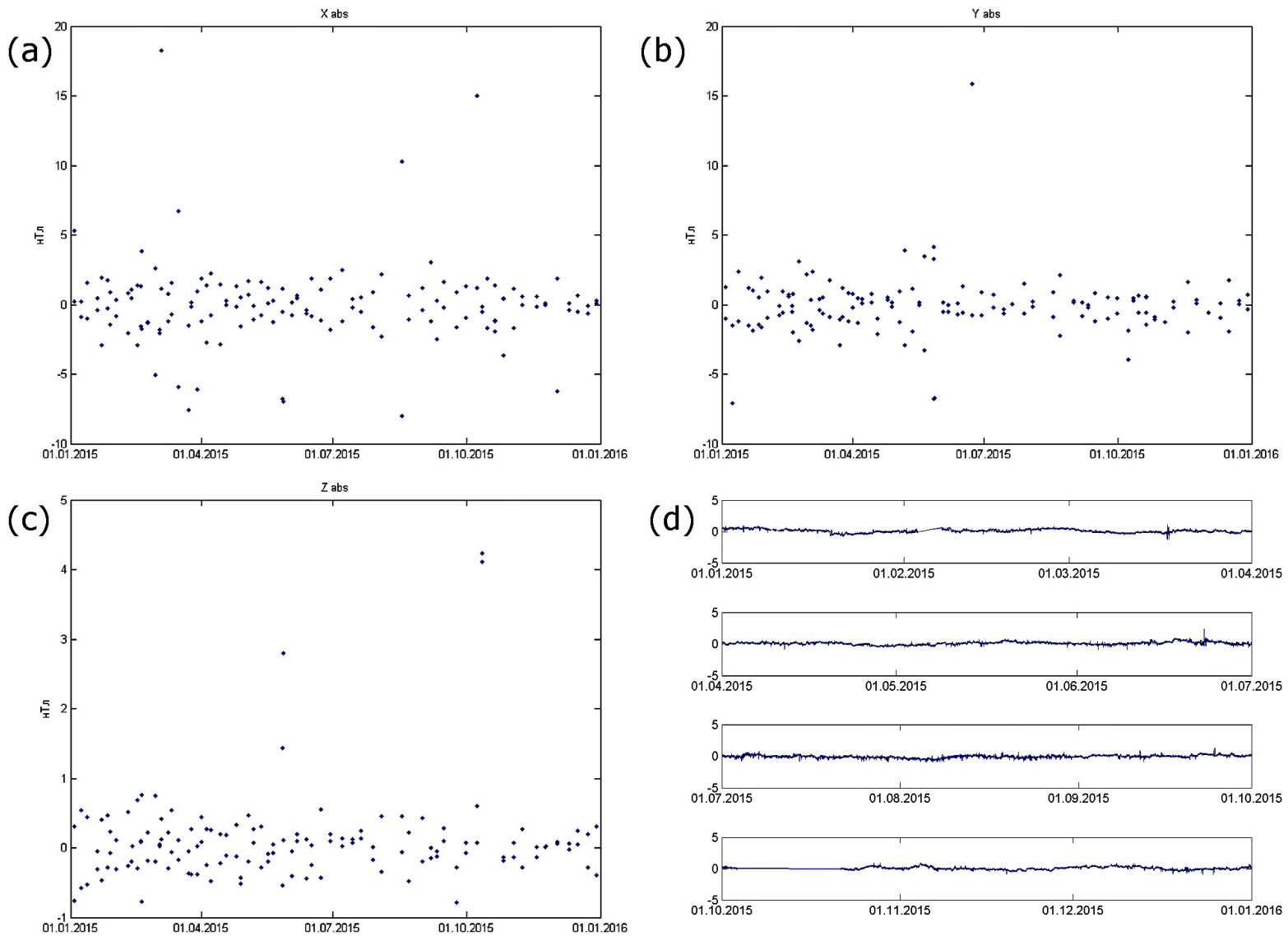

Figure 10. Differences in the absolute measurements of the magnetic field components and definitive 1 min values taken for the corresponding time moments: $X$ component (a), $Y$ component (b), $Z$ component (c), and fragments of the $\Delta F$ record over 2015 (d).

sults of research and can be cited on the same basis as other scientific references.

Data availability. Nearly all the underlying research datasets can be accessed via web. Variation, quasi-definitive, and definitive data for SPG magnetic observatory can be downloaded at the Russian-Ukrainian Geomagnetic Data Center (http://geomag.gcras. $\mathrm{ru}$ /dataprod-down.html) or via www.intermagnet.org. Variation, quasi-definitive and definitive data for SPG magnetic observatory (Soloviev, 2016; Soloviev et al., 2016a, b) can be downloaded. Absolute and baseline values registered at the SPG observatory can be accessed at the page http://geomag.gcras.ru/dataserv-abs.html. The DOI defined for the variation and definitive datasets are included into the reference list. Geomagnetic data from other observatories can also be downloaded from www.intermagnet.org. Unfortunately, the source data recorded during a geomagnetic survey cannot be shared and accessed as they were lost.

Author contributions. The collaboration of the GC RAS and IZMIRAN that led to the Saint Petersburg magnetic observatory installation and development was set up by the joint efforts of the Laboratory of Geoinformatics and Geomagnetic Studies of the GC RAS, headed by AS, and the Department of Geomagnetic Researches of the IZMIRAN SPb, directed by YK.

The magnetic survey of the observatory territory was performed by RS and AG. Magnetic hardware was installed and set up for data registering by nearly all the authors of this pater except DK who later thoroughly controlled the magnetometer setup and monitored possible hardware failures.

Observatory infrastructure renovation, pavilion repair, power supply provision, and azimuth mark construction was possible due to the orders of YK and AS. Observatory hardware and software maintenance and renovation was performed by AK and PS and guided and assisted by AG. All geodetic measurements for pillar coordinate and reference azimuth determination were done by RK.

All absolute measurements at the Saint Petersburg observatory that later were used to calculate the definitive magnetic data and their operational calculation using the web services of the RussianUkrainian Geomagnetic Data Center were performed by AK and PS. AK has been a regular staff member (magnetologist) of the observatory since its installation in 2012. Theodolite handover for its periodic servicing (verification and repair) was assisted by RS and RK.

The variation data preparation, its despiking, as well as baseline and definitive data calculation and their quality control was done by DK. The comparisons between the observatory data and the vari- 
ation data from other observatories and the model data were performed by AS and RS.

Competing interests. The authors declare that they have no conflict of interest.

Acknowledgements. The results presented in this paper rely on data collected at the INTERMAGNET magnetic observatories. We express our gratitude to the national institutes that support them, we are grateful to the INTERMAGNET community for promoting the high standards of magnetic observatory practice (http://www.intermagnet.org) and the Russian-Ukrainian Geomagnetic Data Center (http://geomag.gcras.ru; re3data.org, 2017) for making the data freely available online. The research has been conducted in the framework of the Russian Science Foundation project no. 17-17-01215.

Edited by: Valery Korepanov

Reviewed by: László Hegymegi and one anonymous referee

\section{References}

Benoit, S. L. (Ed.): INTERMAGNET Technical Reference Manual, Version 4.6, INTERMAGNET, Edinburgh, 2012.

Chulliat, A. and Anisimov, S.: The Borok INTERMAGNET magnetic observatory, Russ. J. Earth. Sci., 10, ES3003, https://doi.org/10.2205/2007ES000238, 2008.

Finlay, C. C., Olsen, N., Kotsiaros, S., Gillet, N., and ToeffnerClausen, L.: Recent geomagnetic secular variation from Swarm and ground observatories as estimated in the CHAOS6 geomagnetic field model. Earth Planets Space, 68, 112, https://doi.org/10.1186/s40623-016-0486-1, 2016.

Gvishiani, A., Lukianova, R., Soloviev, A., and Khokhlov, A.: Survey of Geomagnetic Observations Made in the Northern Sector of Russia and New Methods for Analysing Them, Surv. Geophys., 35, 1123-1154, https://doi.org/10.1007/s10712-0149297-8, 2014.

Gvishiani, A., Soloviev, A., Krasnoperov, R., and Lukianova, R.: Automated Hardware and Software System for Monitoring the Earth's Magnetic Environment, Data Science Journal, 15, 1-24, https://doi.org/10.5334/dsj-2016-018, 2016.

Gvishiani, A. D. and Lukianova, R Yu.: Geoinformatics and observations of the Earth's magnetic field: The Russian segment, Izv.-Phys. Solid Earth. 51, 157-175, https://doi.org/10.1134/S1069351315020044, 2015.

Gvishiani, A. D., Sidorov, R. V., Lukianova, R. Yu., and Soloviev, A. A.: Geomagnetic activity during St. Patrick's Day storm inferred from global and local indicators, Russ. J. Earth Sci., 16, ES6007, https://doi.org/10.2205/2016ES000593, 2016.

Hafez, A. and Ghamry, E.: Geomagnetic Sudden Commencement Automatic Detection via MODWT, IEEE T. Geosci. Remote, 51, 1547-1554, https://doi.org/10.1109/TGRS.2012.2207962, 2013.

Jankowski, J. and Sucksdorff, C.: Guide for magnetic measurements and observatory practice, IAGA, Warsaw, 1996.
Kaftan, V. I. and Krasnoperov, R. I.: Geodetic Observations at Geomagnetic Observatories, Geomagn. Aeronomy, 55, 118-123, https://doi.org/10.1134/S0016793215010065, 2015.

Kp Index - Helmholtz Centre Potsdam GFZ German Research Centre for Geosciences: http://www.gfz-potsdam.de/en/section/ earths-magnetic-field/data-products-services/kp-index/, last accessed: 17 February 2017.

Krasnoperov, R. I., Sidorov, R. V., and Soloviev, A. A.: Modern Geodetic Methods for High-Accuracy SurveyCoordination on the Example of Magnetic Exploration, Geomagn. Aeronomy, 55, 547-554, https://doi.org/10.1134/S0016793215040076, 2015.

Lalanne, X., Peltier, A., Chulliat, A., Telali, A., and Heumez, B.: A new measurement method for magnetic repeat stations, in: Proceedings of the XVth IAGA Workshop on Geomagnetic Observatory Instruments, Data Acquisition, and Processing, San Fernando, Cadiz, Spain, 4-14 June 2012, 138-141, 2013.

Magnetic Crustal Field Model - EMM2015: http://ngdc.noaa.gov/ geomag/EMM/, last accessed: 20 March 2017.

Nechaev, S. A.: Guide for stationary geomagnetic observations, V.B. Sochava Institute of Geography SB RAS, Irkutsk, Russia, 2006.

Newitt, L. R., Barton, C. E., and Bitterly, J.: Guide for magnetic repeat station surveys, International Association of Geomagnetism and Aeronomy, Boulder, USA, 1996.

Olsen, N., Hulot, G., Lesur, V., Finlay, C. C., Beggan, C., Chulliat, A., Sabaka, T. J., Floberghagen, R., Friis-Christensen, E., Haagmans, R., Kotsiaros, S., Lühr, H., Tøffner-Clausen, L., and Vigneron, P.: The Swarm Initial Field Model for the 2014 geomagnetic field, Geophys. Res. Lett., 42, 1092-1098, https://doi.org/10.1002/2014GL062659, 2015.

Pasetsky, V. M. and Svetlaev, G. D.: Magnetic and meteorological observatory Pavlovsk-Voeikovo, Hydometeoizdat, Leningrad, USSR, 1978.

re3data.org: Russian-Ukrainian Geomagnetic Data Center; editing status 2015-05-28; re3data.org - Registry of Research Data Repositories, https://doi.org/10.17616/R39344, 2017.

Russian-Ukrainian Geomagnetic Data Center: http://geomag.gcras. ru/, last accessed: 17 February 2017.

Sapunov, V. A., Denisov, A. Y., Saveliev, D. V., Soloviev, A. A., Khomutov, S. Y., Borodin, P. B., Narkhov, E. D., Sergeev, A. V., and Shirokov, A. N.: New vector/scalar Overhauser DNP magnetometers POS-4 for magnetic observatories and directional oil drilling support, Magnetic Resonance in Solids: Electronic Journal, 18, 16209, 2016.

Soloviev, A. (Ed.): Geomagnetic data recorded at Geomagnetic Observatory Saint Petersburg (IAGA code: SPG), ESDB repository, Geophysical Center of the Russian Academy of Sciences, https://doi.org/10.2205/SPG2012, 2016.

Soloviev, A., Bogoutdinov, S., Gvishiani, A., Kulchinskiy, R., and Zlotnicki, J.: Mathematical Tools for Geomagnetic Data Monitoring and the INTERMAGNET Russian Segment, Data Science Journal, 12, WDS114-WDS119, https://doi.org/10.2481/dsj.WDS-019, 2013.

Soloviev, A., Dobrovolsky, M., Kudin, D., and Sidorov, R.: Minute values of $\mathrm{X}, \mathrm{Y}, \mathrm{Z}$ components and total intensity $F$ of the Earth's magnetic field from Geomagnetic Observatory Saint Petersburg (IAGA code: SPG), ESDB repository, Geophysical Center of the Russian Academy of Sciences, https://doi.org/10.2205/SPG2012min, 2016a. 
Soloviev, A., Kopytenko, Y., Kotikov, A., Kudin, D., and Sidorov, R.: 2015 definitive data from geomagnetic observatory Saint Petersburg (IAGA code: SPG): minute values of $\mathrm{X}, \mathrm{Y}, \mathrm{Z}$ components and total intensity F of the Earth's magnetic field, ESDB repository, Geophysical Center of the Russian Academy of Sciences, https://doi.org/10.2205/SPG2015min-def, 2016b.
$\mathrm{Wu}$, J.-G. and Lundstedt, H.: Geomagnetic storm predictions from solar wind data with the use of dynamic neural networks, J. Geophys. Res., 102, 14255-14268, https://doi.org/10.1029/97JA00975, 1997. 